\title{
AN UNBIASED SCATTERING FUNCTION ESTIMATOR FOR FAST TIME-VARYING CHANNELS*
}

\author{
Harold Artés, Gerald Matz, and Franz Hlawatsch \\ Institute of Communications and Radio-Frequency Engineering, Vienna University of Technology \\ Gusshausstrasse 25/389, A-1040 Wien, Austria \\ phone: +43158801 38923, fax: +4315870583, email: hartes@aurora.nt.tuwien.ac.at \\ web: http://www.nt.tuwien.ac.at/dspgroup/time.html
}

\begin{abstract}
We propose an unbiased, computationally efficient estimator of the scattering function that is specifically suited to underspread WSSUS channels. The novel estimator allows for nonideal sounding signals, fast time-variation, and on-line operation during data transmission. Its good performance is assessed by a bias/variance analysis and simulation results.
\end{abstract}

\section{INTRODUCTION}

The scattering function characterizes the second-order statistics of random, linear, time-varying channels obeying the usual WSSUS assumption [1, 2]. An estimate of the scattering function is required or helpful for (e.g.) receiver design [2]-[4], channel estimation [5], and performance analysis [6].

We propose an unbiased and computationally efficient scattering function estimator which, in contrast to existing estimators (e.g. [7]-[9]), exploits the underspread property of WSSUS mobile communications channels $[2,10]$. This allows for nonideal sounding signals, fast time-varying channels, and on-line operation during data transmission.

The paper is organized as follows. Section 2 establishes a discrete-time framework, and Section 3 discusses an averaged periodogram estimator and a related sounding technique. Section 4 presents and studies the novel scattering function estimator and extends it to on-line operation. Simulation results are considered in Section 5.

\section{DISCRETE-TIME LTV CHANNELS}

Linear time-varying (LTV) channels are commonly characterized by their time-varying impule response $h(t, \tau)$ (equivalent baseband channels will be considered throughout). For applicability of digital block processing algorithms such as the FFT, we shall use a discrete-time, finite-length framework that is based on the following assumptions:

1. The input signal $x(t)$ is bandlimited (bandwidth $B$ ).

2. The impulse response $h(t, \tau)$ is bandlimited with respect to $t$ (bandwidth $B_{D}$, i.e., maximum Doppler shift $\pm B_{D}$ ) and with respect to $\tau$ (bandwidth $B$ ), and effectively delay-limited with maximum delay $\tau_{\max }$.

3. We consider the output signal $y(t)$ for $t \in\left[0, t_{\max }\right]$. We then obtain the discrete-time input-output relation [11]

$$
y[n]=\sum_{m=0}^{N_{m}-1} h[n, m] x[n-m], \quad n \in[0, N-1],
$$

where $y[n]=y\left(n / f_{s}\right), x[n]=x\left(n / f_{s}\right)$, and $h[n, m]=$ $\left(1 / f_{s}\right) h\left(n / f_{s}, m / f_{s}\right)$ with the sampling frequency $f_{s}=$ $2\left(B+B_{D}\right) ;$ furthermore $N_{m}=\left[\tau_{\max } f_{s}\right]$ and $N=\left[t_{\max } f_{s}\right\rceil$. In this discrete-time setting, the WSSUS property [1] reads

\footnotetext{
${ }^{*}$ Funding by FWF grants P12228-TEC and P11904-TEC.
}

$$
\mathbb{E}\left\{S[m, l] S^{*}\left[m^{\prime}, l^{\prime}\right]\right\}=C[m, l] \delta\left[m-m^{\prime}\right] \delta\left[l-l^{\prime}\right],
$$

where $\mathbb{E}$ denotes expectation, $S[m, l]=\sum_{n=0}^{N-1} h[n, m]$ $e^{-j 2 \pi l n / N}$ is the (delay-Doppler) spreading function, and $C[m, l]$ is the scattering function [1]. Equivalently,

$$
\mathbb{E}\left\{H[n, k] H^{*}\left[n^{\prime}, k^{\prime}\right]\right\}=R_{H}\left[n-n^{\prime}, k-k^{\prime}\right],
$$

where $H[n, k]=\sum_{m=0}^{N_{m}-1} h[n, m] e^{-j 2 \pi k m / N}$ is the timevarying transfer function $[1]$. We note that $S[m, l] \leftrightarrow H[n, k]$ as well as $C[m, l] \leftrightarrow R_{H}[n, k]$ are related by a $2-\mathrm{D}$ DFT of length $N$. The relations (1) and (2) show that $H[n, k]$ is a stationary $2-\mathrm{D}$ random process with autocorrelation function $R_{H}[n, k]$ and power spectral density $C[m, l] \geq 0$. Hence, estimation of the scattering function is a 2-D spectral estimation problem. Note, however, that realizations of the process $H[n, k]$ (whose spectrum is to be estimated) must themselves be estimated by means of channel sounding.

In our discrete-time setting, the underspread property $[2$, 10] means that the scattering function $C[m, l]$ (and thus, with probability one, also the spreading function $S[m, l])$ is zero outside a region ${ }^{1}\left[0, N_{m}-1\right] \times\left[0, N_{l}-1\right]$ with

$$
N_{m} N_{l} \leq N \text {. }
$$

\section{AVERAGED PERIODOGRAM ESTIMATOR} AND VIRTUAL KAILATH SOUNDING

In this section, we describe some techniques that are related to the novel estimator to be proposed in Section 4 .

Averaged periodogram estimator. A classical spectral estimator is the averaged periodogram estimator, i.e., the squared Fourier transform magnitude averaged over several process realizations [12]. Since the process is $H[n, k]$ and its Fourier transform is $S[m, l]$, the averaged periodogram estimator of the scattering function can be written as

$$
\hat{C}_{\mathrm{AP}}[m, l] \triangleq \frac{1}{L} \sum_{i=1}^{L}\left|\hat{S}_{i}[m, l]\right|^{2},
$$

where the $\hat{S}_{i}[m, l]$ are estimates of $S[m, l]$ based on $L$ different channel soundings. (Repeated channel sounding is no problem since the statistics of a WSSUS channel do not change with time.) Next, we describe an efficient implementation of $\hat{C}_{\mathrm{AP}}[m, l]$ that is valid for underspread channels.

Kailath sounding. The conceptual basis of this implementation is a channel sounding technique proposed by Kailath [13] for continuous-time underspread channels and reformulated here in our discrete-time setting. The sound-

${ }^{1}$ For convenience, we assume one-sided $m$ and $l$ intervals. Other locations of these intervals can easily be accommodated by a simple shift. 
ing signal is an impulse train, i.e., $x[n]=K \delta_{M}[n]=$ $K \sum_{r=0}^{N / M-1} \delta[n-r M]$, where the block length $N$ is chosen an integer multiple of the impulse spacing $M$. Throughout this paper, we assume $N_{m} \leq M \leq N / N_{i}$ which presupposes the underspread property (3). Including zero-mean, white measurement noise $w[n]$ with variance $N_{0}$, the resulting output signal is obtained as

$$
\begin{aligned}
y[n] & =K \sum_{r=0}^{N / M-1} h[n, n-r M]+w[n] \\
& =K h\left[n, n-\left\lfloor\frac{n}{M}\right\rfloor M\right]+w[n],
\end{aligned}
$$

where the last expression holds since (due to $M \geq N_{m}$ ) the responses to the individual impulses do not overlap. Thus, $y[n]$ is a noisy, undersampled version of the impulse response. Because of the underspread property, the undersampling does not imply an information loss.

Implementation using the Zak transform. The discrete Zak transform (with time period parameter $M$ ) [14] of the output signal $y[n]$ in (5) is given by [15]

$$
\begin{aligned}
Z_{y}[m, l] & \triangleq \sum_{r=0}^{N / M-1} y[m+r M] e^{-j 2 \pi \frac{r l}{N / M}} \\
& =K \sum_{r=0}^{N / M-1} h[m+r M, m] e^{-j 2 \pi \frac{r l}{N / M}}+Z_{w}[m, l] \\
& =\frac{K}{M} S[m, l] e^{j 2 \pi \frac{m l}{N}}+Z_{w}[m, l]
\end{aligned}
$$

for $(m, l) \in \mathcal{R} \triangleq[0, M-1] \times\left[0, \frac{N}{M}-1\right]$. With $N_{m} \leq M \leq$ $N / N_{l}$, this region $\mathcal{R}$ contains the spreading function support $\left[0, N_{m}-1\right] \times\left[0, N_{l}-1\right]$. Hence, $Z_{y}[m, l]$ equals the spreading function $S[m, l]$ (on its support) up to an amplitude-phase factor and a noise term. This suggests to replace in (4) $\hat{S}_{i}\{m, l]$ with $\frac{M}{K} Z_{y_{i}}\{m, l] e^{-j 2 \pi \frac{m l l}{N}}$, where $y_{i}[n]$ is the output signal obtained by the $i$ th Kailath sounding. This yields the following efficient implementation of $\hat{C}_{\mathrm{AP}}[m, l]$ :

$$
\hat{C}_{\mathrm{AP}}\{m, l]=\frac{1}{L}\left(\frac{M}{K}\right)^{2} \sum_{i=1}^{L}\left|Z_{y_{i}}[m, l]\right|^{2}, \quad(m, l) \in \mathcal{R},
$$

with $Z_{y_{i}}[m, l]=\sum_{r=0}^{N / M-1} y_{i}[m+r M] e^{-j 2 \pi \frac{r l}{N / M}}$.

Virtual Kailath sounding. Due to its high crest factor, an impulse-train sounding signal is undesirable in practice. Instead, we can transmit a pulse-train sounding signal $x[n]=\sum_{r=0}^{N / M-1} g[n-r M]$ with low crest factor and use pulse compression by means of a receive filter $f[n][16]$.

Let $\mathbf{H}$ denote the LTV channel filter. The output of the receive filter is

$$
y_{f}[n] \triangleq(y * f)[n]=((\mathbf{H} x) * f)[n]+w_{f}[n],
$$

where $w_{f}[n]=(w * f)[n]$ is the filtered noise. As we will show presently, if the product of maximum Doppler shift $N_{l}$ and length of receive filter $N_{f}$ satisfies $N_{l} N_{f} \ll N$, then the LTV channel filter $\mathbf{H}$ and the receive filter $f$ approximately commute, i.e., $((\mathbf{H} x) * f)[n] \approx(\mathbf{H}(x * f))[n]=\left(\mathbf{H} x_{v}\right)[n]$ for all $x[n]$, with the virtual sounding signal $x_{v}[n] \triangleq(x * f)[n]$ (note that conceptually, we have moved the receive filter to the transmitter side). Hence,

$$
y_{f}[n] \approx\left(\mathbf{H} x_{v}\right)[n]+w_{f}[n] \triangleq y_{v}[n] .
$$

The $L_{1}$ norm of the approximation error, $\left\|y_{f}-y_{v}\right\|_{1}=$ $\sum_{n=0}^{N-1}\left|y_{f}[n]-y_{v}[n]\right|$, can be shown to be bounded as

$$
\frac{\left\|y_{f}-y_{v}\right\|_{1}}{|x[n]|_{\max }\|S\|_{1}\|f\|_{1}} \leq 2 \pi \frac{N_{l} N_{f}}{N}
$$

with $\|S\|_{1}=\sum_{m=0}^{M-1} \sum_{l=0}^{N / M-1}|S[m, l]|$ and $\|f\|_{1}=\sum_{n=0}^{N_{f}-1}|f[n]|$. Hence, $y_{f}[n] \approx y_{v}[n]$ for $N_{l} N_{f} \ll N$.

For $x[n]=\sum_{r=0}^{N / M-1} g[n-r M]$, the virtual sounding signal

$$
x_{v}[n]=\sum_{r=0}^{N / M-1}(g * f)[n-r M] .
$$

Here, $g[n]$ and $f[n]$ must be chosen such that $(g * f)[n]$ is impulse-like and hence $x_{v}[n]$ is approximately an impulse train. In particular, this will be the case if $g[n]$ is a maximum-length pseudonoise sequence and $f[n]=g^{*}[-n]$, so that $(g * f)[n]$ becomes the autocorrelation of $g[n]$.

\section{THE NOVEL ESTIMATOR}

Application of virtual Kailath sounding to fast time-varying channels requires a small receive filter length $N_{f}$ to keep the "commutation error" (8) small. However, a small $N_{f}$ results in poor pulse compression and, hence, systematic sounding errors. Even larger sounding errors may be encountered with on-line estimation during data transmission (see further below). We now propose a scattering function estimator that compensates for non-perfect virtual sounding signals.

Motivation. Let $A_{s}[m, l] \triangleq \sum_{n=0}^{N-1} s[n] s^{*}[n-m] e^{-j 2 \pi \frac{n l}{N}}$ denote the (cyclic) discrete ambiguity function of a length$N$ signal $s[n]$ and let $\bar{A}_{s}[m, l] \triangleq \mathbb{E}\left\{A_{s}[m, l]\right\}$. Using $y_{v}[n]=$ $\left(\mathbf{H} x_{v}\right)[n]+w_{f}[n]$ from $(7)$, one can show (cf. [7])

$$
\bar{A}_{y_{v}}[m, l]=R_{H}[m, l] A_{x_{v}}[m, l]+\bar{A}_{w_{f}}[m, l],
$$

where $\bar{A}_{w_{f}}[m, l]=N N_{0} r_{f}[m] \delta[l]$ with $r_{f}[m]=\sum_{n=0}^{N_{f}-1} f[n]$ $f^{*}[n-m]$. Assuming approximate commutation of channel and receive filter, so that $y_{v}[n] \approx y_{f}[n]$ according to (7), relation (9) suggests the following estimator of $R_{H}[n, k]$,

$\hat{R}_{H}[n, k] \triangleq \frac{\hat{A}_{y_{f}}[n, k]-\bar{A}_{w_{f}}[n, k]}{A_{x_{v}}[n, k]}=\frac{\hat{A}_{y_{f}}[n, k]-N N_{0} r_{f}[n] \delta[k]}{A_{x_{v}}[n, k]}$

where

$$
\hat{A}_{y_{f}}[n, k]=\frac{1}{L} \sum_{i=1}^{L} A_{y_{f, i}}[n, k]
$$

is an estimate of $\bar{A}_{y_{f}}[n, k]$ that uses $L$ filtered channel output signals $y_{f, i}[n]$ obtained by repeated virtual Kailath sounding; furthermore, it is assumed that $N_{0}$ is known or has been estimated. From $\hat{R}_{H}[n, k]$, a scattering function estimator can be derived by a 2-D DFT. A serious problem, however, is the impossibility of finding an $x_{v}[n]$ for which $\left\{A_{x_{v}}[n, k]\right\}$ is sufficiently bounded away from zero for all $n$ and $k$.

"Subsampled" estimator. This problem can be circumvented since the channel is underspread, i.e., $C[m, l]=0$ outside $\mathcal{R}$. This implies a bandlimited $R_{H}[n, k]$ that can be reconstructed without error from the subsampled version $R_{H}\left[r M, s \frac{N}{M}\right]$. We are thus led to replacing (10) by

$$
\hat{R}_{H}\left[r M, s \frac{N}{M}\right]=\frac{\hat{A}_{y_{f}}\left[r M, s \frac{N}{M}\right]-N N_{0} r_{f}[r M] \delta[s]}{A_{x_{v}}\left[r M, s \frac{N}{M}\right]}
$$


with $r=0,1, \cdots, \frac{N}{M}-1, s=0,1, \cdots, M-1$. Here, $\left|A_{x_{v}}[n, k]\right|$ need only be sufficiently bounded away from zero for $n=$ $r M$ and $k=s \frac{N}{M}$. It can be shown that $\hat{A}_{y_{f}}\left[r M, s \frac{N}{M}\right]=$ $\frac{1}{L} \sum_{i=1}^{L} A_{y_{f, i}}\left[r M, s \frac{N}{M}\right]$ can be efficiently computed as

$$
\hat{A}_{y_{f}}\left[r M, s \frac{N}{M}\right]=\frac{M}{N} \sum_{m=0}^{M-1} \sum_{l=0}^{N / M-1} z[m, l] e^{j 2 \pi\left(\frac{l r}{N / M}-\frac{m s}{M}\right)},
$$

with $z[m, l] \triangleq \frac{1}{L} \sum_{i=1}^{L}\left|Z_{y_{f, i}}[m, l]\right|^{2}$.

From $\hat{R}_{H}\left[r M, s \frac{N}{M}\right]$ in (11), the proposed scattering function estimator is finally obtained via a 2-D DFT,

$$
\hat{C}[m, l]=N \sum_{r=0}^{N / M-1} \sum_{s=0}^{M-1} \hat{R}_{H}\left[r M, s \frac{N}{M}\right] e^{-j 2 \pi\left(\frac{l r}{N / M}-\frac{m s}{M}\right)} .
$$

If $N_{f} \leq M$, then $r_{f}[r M]=E_{f} \delta[r]$ with $E_{f}=r_{f}[0]$. Here, (13) becomes

$$
\hat{C}[m, l]=N \sum_{r=0}^{N / M-1} \sum_{s=0}^{M-1} \frac{\hat{A}_{y_{f}}\left[r M, s \frac{N}{M}\right]}{A_{x_{v}}\left[r M, s \frac{N}{M}\right]} e^{-j 2 \pi\left(\frac{l r}{N / M}-\frac{m s}{M}\right)}-\gamma
$$

where $\gamma=N^{2} N_{0} E_{f} / E_{x_{v}}$ with $E_{x_{v}}=\sum_{n=0}^{N-1}\left|x_{v}[n]\right|^{2}=A_{x_{v}}[0,0]$. This estimator is computationally efficient-its complexity is $\mathcal{O}(N[(L+2) \log (N / M)+2 \log M])$, with substantial additional savings existing for $N_{m} N_{l} \ll N$ (cf. Section 5).

Relation to the periodogram estimator. The ideal virtual sounding signal $x_{v}[n]$ is the impulse train $K \delta_{M}[n]=$ $K \sum_{r=0}^{N / M-1} \delta[n-r M]$ since it is the only signal whose subsampled ambiguity function is constant, $A_{K \delta_{M}}\left[r M, s \frac{N}{M}\right] \equiv$ $K^{2} \frac{N}{M}$. Substituting this for $A_{x_{v}}\left[r M, s \frac{N}{M}\right]$ in (14) and omitting the noise bias correction $\gamma$, we obtain with (12)

$$
\hat{C}[m, l]=\frac{M^{2}}{K^{2}} \frac{1}{L} \sum_{i=1}^{L}\left|Z_{y_{f, i}}[m, l]\right|^{2} \quad \text { for } x_{v}[n]=K \delta_{M}[n] .
$$

This is equivalent to $\hat{C}_{\mathrm{AP}}[m, l]$ in (6). Thus, the averaged periodogram estimator is a special case of our proposed estimator using an idealized impulse train as virtual sounding signal and no noise bias correction. In this light, the division by $A_{x_{v}}\left[r M, s \frac{N}{M}\right]$ in (11) can be viewed as an "equalization" that allows for nonideal virtual sounding signals.

Bias/variance analysis. We now consider the bias and variance of the proposed estimator $\hat{C}[m, l]$ in (13), (11). We assume perfect commutation of channel and receive filter, so that $y_{f}[n]=y_{v}[n]$. With (9) and $\mathbb{E}\left\{\hat{A}_{y_{f}}[m, l]\right\}=\bar{A}_{y_{f}}[m, l]=$ $\bar{A}_{y_{v}}[m, l]$, it easily follows that the estimator is unbiased, i.e., $\mathbb{E}\{\hat{C}[m, l]\} \equiv C[m, l]$. This can be attributed to the noise bias correction and "equalization" in (11). Let us compare this to the averaged periodogram estimator (6) using virtual Kailath sounding and noise bias correction (cf. (14)),

$$
\hat{C}_{\mathrm{APN}}[m, l] \triangleq \frac{1}{L} \frac{N M}{E_{x_{v}}} \sum_{i=1}^{L}\left|Z_{y_{f, i}}[m, l]\right|^{2}-\gamma .
$$

It can be shown that

$$
\mathbb{E}\left\{\hat{C}_{\mathrm{APN}}\{m, l]\right\}=\frac{1}{N E_{x_{v}}} \sum_{r=0}^{N / M-1} \sum_{s=0}^{M-1} \tilde{C}\left[m-r M, l-s \frac{N}{M}\right],
$$

where $\widetilde{C}[m, l] \triangleq \sum_{n=0}^{N-1} \sum_{k=0}^{N-1} C[m-n, l-k] W_{x_{v}}[n, k]$ with
$W_{x_{v}}[n, k] \triangleq \frac{1}{N} \sum_{m=0}^{N-1} \sum_{l=0}^{N-1} A_{x_{v}}[m, l] e^{j 2 \pi \frac{n l-k m}{N}}$. Hence, the averaged periodogram estimator is biased even if we include noise bias correction.

For variance calculation, we assume $w[n]$ and $h[n, m]$ to be circularly symmetric complex Gaussian and all channel soundings to be separated by at least the channel's coherence time [2] so that the $y_{f, i}[n]$ are statistically independent. The mean variance of our estimator can then be shown to be

$$
\begin{aligned}
V & \triangleq \frac{1}{N} \sum_{m=0}^{M-1} \sum_{l=0}^{N / M-1} \operatorname{var}\{\hat{C}[m, l]\} \\
& =\frac{N}{L} \sum_{r=0}^{N / M-1} \sum_{s=0}^{M-1} \sum_{n=0}^{N-1} \sum_{k=0}^{N-1} \frac{\left|\bar{A}_{y_{f}}[n, k]\right|^{2}}{\left|A_{x_{v}}\left[r M, s \frac{N}{M}\right]\right|^{2}} e^{-j 2 \pi\left(\frac{n s}{M}-\frac{k r}{N / M}\right)} .
\end{aligned}
$$

Inserting $y_{f}[n]=y_{v}[n]=\sum_{m=0}^{N_{m}-1} h[n, m] x_{v}[n-m]+w_{f}[n]$, $V$ can be expressed in terms of $C[m, l], A_{x_{v}}[n, k]$, and $P_{w_{f}}[k]=N_{0}|F[k]|^{2}$ (the power spectral density of $w_{f}[n]$ ). For simplicity, we only present the upper bound

where

$$
V \leq \frac{\beta}{L} N \sum_{r=0}^{N / M-1} \sum_{s=0}^{M-1} \frac{1}{\left|A_{x_{v}}\left[r M, s \frac{N}{M}\right]\right|^{2}}
$$

$$
\beta \triangleq E_{x_{v}}^{2} R_{H}^{2}[0,0]+2 N E_{x_{v}} R_{H}[0,0]\left[P_{w_{f}}[k]\right]_{\max }+N\left\|P_{w_{f}}\right\|^{2}
$$

with $R_{H}[0,0]=\frac{1}{N^{2}} \sum_{m=0}^{M-1} \sum_{l=0}^{N / M-1} C[m, l]$ (the average path loss [2]) and $\left\|P_{w_{f}}\right\|^{2}=\sum_{k=0}^{N-1} P_{w_{f}}^{2}[k]$. According to (16), the variance may be high if $\left\{A_{x_{v}}\left[r M, s \frac{N}{M}\right]\right\}$ contains small values. For comparison, the mean variance of $\hat{C}_{\mathrm{APN}}[m, l]$ in (15) is bounded as

$$
V_{\text {APN }} \leq \frac{\beta}{L} \frac{N^{2}}{E_{x_{v}}^{2}}
$$

Since $\left|A_{x_{v}}\left[r M, s \frac{N}{M}\right]\right|^{2} \leq E_{x_{v}}^{2}$, the bound on $V$ in (16) is always $\geq$ the bound on $V_{\text {APN }}$ in (17), with equality if and only if $\bar{x}_{v}[n]=K \delta_{M}[n]$ (where $\left|A_{x_{v}}\left[r M, s \frac{N}{M}\right]\right| \equiv E_{x_{v}}$ ).

In conclusion, the "equalization" causes our estimator to be unbiased for any $x_{v}[n]$ but it may result in a larger variance if $x_{v}[n] \neq K \delta_{M}[n]$. However, the variance can always be decreased by more averaging (i.e., a larger $L$ ) whereas a nonzero bias can never be decreased (cf. Section 5).

On-line estimation. Finally, we outline an extension of our scattering function estimator to on-line operation during data transmission (see [17] for details). Here, the sounding signal $x[n]$ is a segment of the data signal which is assumed known (since the data have been detected). In general, this data signal will not generate an appropriate virtual sounding signal. Therefore, we replace the receive filter $f[n]$ by a bank of $N / M$ receive filters $f_{r}[n](r=1, \cdots, N / M)$, where $f_{r}[n]$ is matched to the $r$ th length- $M$ block of the input signal, i.e., $f_{r}[n]=x^{*}[r M-n]$ for $n=0,1, \cdots, M-1$. The overall output signal is formed as

$$
y_{f}[n] \triangleq \sum_{r=0}^{N / M-1} y_{r}[n]
$$

where $y_{r}[n] \triangleq y_{f_{r}}[n] \operatorname{rect}_{M}[n-r M]=\left(y * f_{r}\right)[n] \operatorname{rect}_{M}[n-$ $r M]$ is the windowed output of the $r$ th receive filter (here, $\operatorname{rect}_{M}[n]$ denotes the rectangular window with support $[0, M-1])$. Assuming suitable correlation properties of the data signal $x[n]$ (which typically hold for CDMA and OFDM signals), it can be argued [17] that this approach approximates an impulse-train virtual sounding signal. 


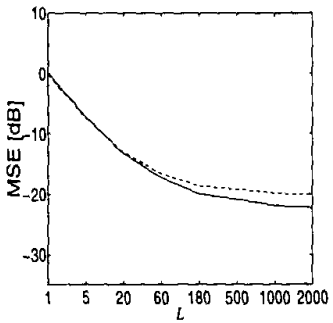

(a)

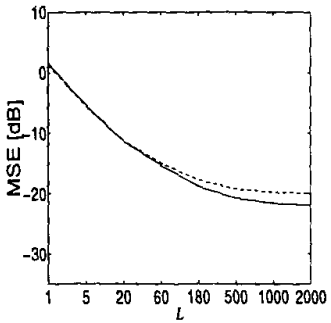

(b)
Figure 1. Comparison of MSEs $\varepsilon_{\hat{C}}$ (solid line) and $\varepsilon_{\hat{C}_{A P N}}$ (dotted line) as a function of $L$ for matched receive filter $\left(N_{f}=31\right)$ : (a) no noise, (b) $S N R=0 d B$.

\section{SIMULATION RESULTS}

In order to assess the performance of our estimator, we simulated a random time-varying channel that corresponds to a system operating at carrier frequency $1.8 \mathrm{GHz}$ with maximurn Doppler shift $B_{D}=100 \mathrm{~Hz}$ (corresponding to maximum velocity $60 \mathrm{~km} / \mathrm{h}$ ) and maximum delay spread $\tau_{\max }=7 \mu \mathrm{s}$. We assumed a sampling frequency of $f_{s}=$ $1 \mathrm{MHz}$ and a sounding duration of about $150 \mathrm{~ms}$, resulting in $N=149358, N_{m}=7$, and $N_{l}=15$. The sounding pulse $g[n]$ was chosen as a maximum-length pseudonoise sequence of length $M=31$, corresponding to a duration of $31 \mu \mathrm{s}$. Since $N_{m} N_{l}=105 \ll N$, we were able to implement a subsampled version of our estimator in which the subsampled ambiguity function (cf. Section 4) was once again subsampled.

In the following, $\varepsilon_{\hat{C}}$ and $\varepsilon_{\hat{C}_{\mathrm{APN}}}$ will denote the (estimated) mean-square error (MSE) of $\hat{C}[m, l]$ and $\hat{C}_{\mathrm{APN}}[m, l]$, respectively, summed over $m$ and $l$ and normalized by $\|C\|^{2}$. We note that the theoretical MSE is the sum of the squared bias and the variance, and we recall that $\hat{C}[m, l]$ is unbiased (hence, $\varepsilon_{\hat{C}}$ theoretically equals the variance). Fig. $1 \mathrm{com}$ pares $\varepsilon_{\hat{C}}$ and $\varepsilon_{\hat{C}_{\mathrm{APN}}}$ as a function of $L$ (the number of soundings used to calculate the estimators), both for the case of no noise and for an SNR of $0 \mathrm{~dB}$ (i.e., $\frac{1}{N} \sum_{n=0}^{N-1} \mathbb{E}\left\{\left|y_{f}[n]\right|^{2}\right\}=$ $\left.E_{f} N_{0}\right)$. The receive filter $f[n]$ is matched to the length-31 sounding pulse $g[n]$. It is seen that for small values of $L$, $\varepsilon_{\hat{C}}$ is slightly larger than $\varepsilon_{\hat{C}_{\mathrm{APN}}}$, thus indicating that $\hat{C}[m, l]$ has a larger variance than $\hat{C}_{\mathrm{APN}}[m, l]$. However, for larger $L$ where the variances have already been reduced by averaging, the unbiasedness of $\hat{C}[m, l]$ dominates, causing $\varepsilon_{\hat{C}}$ to be smaller than $\varepsilon_{\hat{C}_{\mathrm{APN}}}$. The decay of $\varepsilon_{\hat{C}}$ and $\varepsilon_{\hat{C}_{\mathrm{APN}}}$ is initially according to $1 / L$. However, for larger values of $L$ both $\varepsilon_{\hat{C}}$ and $\varepsilon_{\hat{C}_{\mathrm{APN}}}$ level off. The main reason for this behavior (besides the nonzero bias in $\varepsilon_{\hat{C}_{\mathrm{APN}}}$ ) is a noticeable commutation error (cf. (8)) that increases the MSE of both estimators.

To reduce the commutation error, we next truncated the receive filter such that $N_{f}=7 \approx M / 4$. The resulting MSE curves are shown in Fig. 2. Whereas the filter truncation decreases the commutation error by a factor of approximately four according to (8), it also deteriorates the virtual sounding signal $x_{v}[n]$. This causes $\hat{C}_{\mathrm{APN}}[m, l]$ to have a large bias and hence a large MSE which is approximately constant. In contrast, the "equalization" inherent in $\hat{C}[m, l]$ compensates for the nonideal sounding signal, resulting in unbiasedness and thus a decay of $\varepsilon_{\hat{C}}$ according to $1 / L$. Hence, for values of $L$ that are not too small, such that the variance is sufficiently decreased, $\varepsilon_{\hat{C}}$ is much smaller than $\varepsilon_{\hat{C}_{\text {APN }}}$.

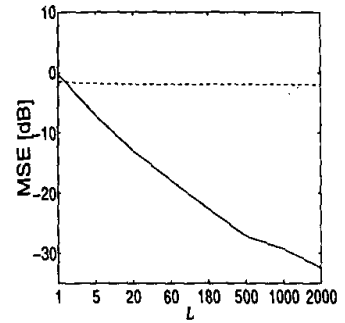

(a)

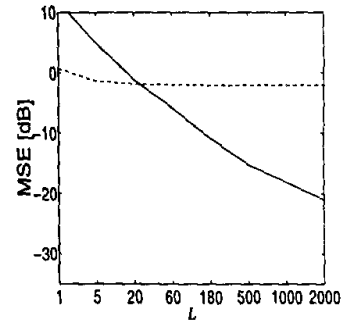

(b)
Figure 2. Comparison of MSEs $\varepsilon_{\hat{C}}$ (solid line) and $\varepsilon_{\hat{C}_{A P N}}$ (dotted line) as a function of $L$ for truncated receive filter $\left(N_{f}=7\right)$ : (a) no noise, (b) $S N R=0 d B$.

\section{REFERENCES}

[1] P. A. Bello, "Characterization of randomly time-variant linear channels," IEEE Trans. Comm. Syst., vol. 11, pp. 360393, 1963.

[2] R. S. Kennedy, Fading Dispersive Communication Channels. New York: Wiley, 1969.

[3] W. Kozek and A. F. Molisch, "Nonorthogonal pulseshapes for multicarrier communications in doubly dispersive channels," IEEE J. Sel. Areas Comm., vol. 16, pp. 1579-1589, Oct. 1998.

[4] A. M. Sayeed and B. Aazhang, "Joint multipath-Doppler diversity in mobile wireless communications," IEEE Trans. Comm., vol. 47, pp. 123-132, Jan. 1999.

[5] Y. Li, L. Cimini, and N. Sollenberger, "Robust channel estimation for OFDM systems with rapid dispersive fading channels," IEEE Thans. Comm., vol. 46, pp. 902-915, July 1998.

[6] F. Adachi and J. D. Parsons, "Error rate performance of digital FM mobile radio with postdetection diversity," IEEE Trans. Comm., vol. 37, pp. 200-210, 1989.

[7] N. T. Gaarder, "Scattering function estimation," IEEE Trans. Inf. Theory, vol. 14, no. 5, pp. 684-693, 1968.

[8] D. W. Ricker and M. J. Gustafson, "A low sidelobe technique for the direct measurement of scattering functions," IEEE $J$. of Oceanic Engineering, vol. 21, pp. 14-23, Jan. 1996.

[9] S. M. Kay, "Estimation of the range-Doppler scattering function based on autoregressive modeling," tech. rep., June 1993.

[10] J. G. Proakis, Digital Communications. New York: McGrawHill, 3rd ed., 1995.

[11] H. Artés, G. Matz, and F. Hlawatsch, "Linear time-varying channels," tech. rep., Institute of Communications and Radio-Frequency Engineering, Vienna University of Technology, 1999.

[12] S. M. Kay, Modern Spectral Estimation. Englewood Cliffs (NJ): Prentice Hall, 1988.

[13] T. Kailath, "Measurements on time-variant communication channels," IEEE Trans. Inf. Theory, vol. 8, pp. 229-236, Sept. 1962.

[14] H. Bölcskei and F. Hlawatsch, "Discrete Zak transforms polyphase transforms, and applications," IEEE Trans. Signal Processing, vol. 45, pp. 851-866, April 1997.

[15] W. Kozek and H. G. Feichtinger, "Time-frequency structured decorrelation of speech signals via nonseparable Gabor frames," in Proc. IEEE ICASSP-97, (Munich, Germany), pp. 1439-1442, April 1997.

[16] J. D. Parsons, D. A. Demery, and A. M. D. Turkmani, "Sounding techniques for wideband mobile radio channels: a review," Prac. IEE-I, vol. 138, pp. 437-446, Oct. 1991.

[17] H. Artés, G. Matz, and F. Hlawatsch, "Unbiased scattering function estimation during data transmission," in Proc. IEEE VTC-99/Fall, (Amsterdam, The Netherlands), Sept. 1999. submitted. 